\title{
Investigation of 3G radio network topologies considering performance and exposure aspects
}

\author{
H. Buddendick ${ }^{1}$, P. Wertz ${ }^{1}$, T. F. Eibert ${ }^{1}$, F. M. Landstorfer ${ }^{1}$, and G. Wölfle ${ }^{2}$ \\ ${ }^{1}$ Institute of Radio Frequency Technology, Universität Stuttgart, 70550 Stuttgart, Germany \\ ${ }^{2}$ AWE Communications GmbH, Otto-Lilienthal-Str. 36, 71034 Böblingen, Germany
}

\begin{abstract}
Five different radio network topologies, in the following referred to as "scenario", have been analyzed with regard to their capability to cover a medium size city with $3 \mathrm{G}$ mobile services. T-Mobile launched this study to assess different radio network topologies in terms of exposure. Thus, the main focus is to examine if, for exposure reasons, a medium size city can be covered with outer-city located sites.

The radio network topologies discussed in this paper can generally be divided into two categories: In category one, the sites are located in the city, and in the other, the sites are located outside of the built-up area. In both cases the number of sites per scenario varies, and additionally the antenna height and site distance to the built-up area vary for the second category.

The investigations are performed with a ray optical propagation model based on 3-D building data and a dynamic 3G network simulator. A mix of four services and a realistic spatial traffic distribution based on land usage is assumed.

The performance of the scenarios, assessed by the evaluation of coverage and capacity, is examined together with the exposure.

It is shown that network topologies with sites located only outside the city are not an adequate solution due to capacity constraints and coverage problems with high data services. A promising network topology is a reduced set of inner-city sites with higher order sectorization.
\end{abstract}

\section{Introduction}

In recent years, the number of people using mobile voice services increased significantly and the availability of mobile communications is taken for granted by most people in in- dustrialized countries. In addition, mobile data services, like web browsing and email, become more and more popular.

In order to provide high capacity radio networks which also offer high bit rate data services for a large percentage of the population, network operators have to redesign their network structure. A straight-forward way, which is preferred by many operators, is to increase the number of base stations and to reduce their distance.

However, if new base stations are to be installed, the network operators are often faced with strong resistance of the local public, since people are afraid of an impact on their health. T-Mobile, like other network operators, takes the people's concerns seriously and is interested to roll-out and extend the network in a widely accepted way. Since it is expected that critical people will probably rather accept alternative network solutions suggested from a third, independent party, T-Mobile launched the study treated in this paper.

This study examines several network topologies to find promising candidates to cover a typical medium size middle European city with mobile services and to fulfill the requirements regarding network performance, economic efficiency and exposure issues. The focus is mainly on UMTS data services, as they are the most crucial ones regarding service coverage. Dynamic system simulations are used to evaluate and compare the above mentioned properties of different network topologies.

The simulation tools (propagation model and 3G system simulator), the considered environment and the investigated network topologies are described in Sect. 2. The results in terms of coverage, capacity and exposure are presented and compared in Sect. 3. Finally Sect. 3 sums up the results.

Correspondence to: H. Buddendick

(buddendick@ihf.uni-stuttgart.de)

Published by Copernicus Publications on behalf of the URSI Landesausschuss in der Bundesrepublik Deutschland e.V. 


\section{Simulation environment and assumptions}

\subsection{Propagation model}

For radio network planning, the prediction of the mobile propagation channel between the sector antennas and the user location in terms of path loss is the most important input parameter. Empirical propagation models only provide limited accuracy in urban environments. On the other hand, ray optical propagation models deliver much more accurate results (Rautiainen et al., 2002). However, ray tracing models have a high computational demand (Zhang et al., 2000). Therefore, a semi-deterministic propagation model based on dominant paths (Wahl et al. , 2005) was utilized in this study.

The main idea of this Urban Dominant Path model (UDP) is that only a few paths contribute a considerable amount of energy to the received power, and that only these paths have to be considered. The physical effects of reflections and diffractions resulting in the typical wave guiding and shadowing phenomena are taken into account.

In comparison to rigorous 3-D ray tracing models the UDP model is much faster but yields comparably prediction accuracy. For a carrier frequency close to the UMTS downlink frequency, a measurement campaign was carried out in the frame of the EU funded MAESTRO project Heyn et al. (2005). The UDP model was validated with these measurement results, yielding standard deviations of the prediction error from $3.5 \mathrm{~dB}$ to $5.6 \mathrm{~dB}$ for different measurement routes.

For mobile stations that are located inside buildings a semi-empirical penetration model is applied.

\subsection{Dynamic 3G system simulator}

It is well known that WCDMA based 3G mobile networks in contrast to GSM networks - show some quite dynamic performance characteristics. In addition, coverage and capacity of the network are strongly correlated (e.g. cell breathing). A system simulation tool has to account for these characteristics. In the same way, a meaningful investigation of exposure aspects has to deal with realistic network loads and the resulting transmission power levels.

One possibility is to perform a dynamic $3 \mathrm{G}$ system simulation. The main drawback of dynamic simulations is the high computational effort. But for general studies and detailed simulations within limited areas, like for the investigations described in this paper, a 3G dynamic simulation tool is well suited and the computation times are in an acceptable range.

The tool used within these studies (Wertz et al., 2002) splits the simulation process into two parts: a dynamic simulation and a scan-mobile process. In the dynamic simulation the network evaluation is performed with mobile stations generated according to statistically defined arrival and serving processes. Thereby the spatial user distribution can be controlled by user densities that depend on the land usage data. Both indoor and outdoor users are generated.
For newly generated mobile stations a check is performed whether the available resources in terms of spreading codes and transmission power is sufficient to fulfill the respective service requirements. If not enough resources are available, or in case of bad pilot coverage, the mobile station is blocked. Fast power control is modeled on UMTS slot basis. If the power control is unable to ensure the required signal to noise ratio target for a given period of time, the mobile station is dropped. The UMTS soft/softer handover procedure is simulated in detail.

In the downlink the imperfect orthogonality due to multipath propagation effects is considered using an orthogonality factor. In accordance with T-Mobile an average orthogonality factor $\alpha=0.75$ was used in this study.

Several statistical outputs are collected in the dynamic simulation. These are mainly the cell and network throughput and the blocking and dropping rates, as well as transmission power and code utilization statistics.

After a certain simulation time the dynamic simulation is interrupted and a probe-mobile scans the complete simulation area in order to provide coverage information (see Fig. 2 and Fig. 3 in Sect. 3.1). The main inputs to the probe-mobile process from the dynamic simulation are instantaneous uplink load and downlink transmission power levels. Both describe the interference conditions for uplink and downlink, respectively.

The coverage is determined for the complete simulation area for several points in time. Considering all time instances, the coverage probability is determined per pixel as the ratio between successful coverage tests and the total number of tests. These tests represent a quasi-static evaluation based on the fulfillment of the $E_{b} / N_{0}$-targets with the defined maximum link power and the evaluation of the CPICH $C / I$. However, they do not account for available spreading code resources. Therefore the coverage plots mainly show the area in which the given traffic density can be distributed.

\subsection{Simulation area and network topologies}

The selected simulation area should include a medium size city and fulfill two demands: first, the terrain should be flat since any effects caused by topographic undulations should be avoided for our investigations. Second, 3-D building data should be available in order to reach the best accuracy of the wave propagation model.

However, 3-D building data in suitable quality are only available for large cities. Thus, we selected a district of Bonn, Germany. This district, called Beuel, was formerly an individual city and kept its old city structure with a small city center, industrial area and residential area.

The land usage types of Beuel and the surrounding area is shown in Fig. 1. The urban area considered in the investigations, corresponding to the area of the former individual city of Beuel, is located within the black polygon. To simulate 


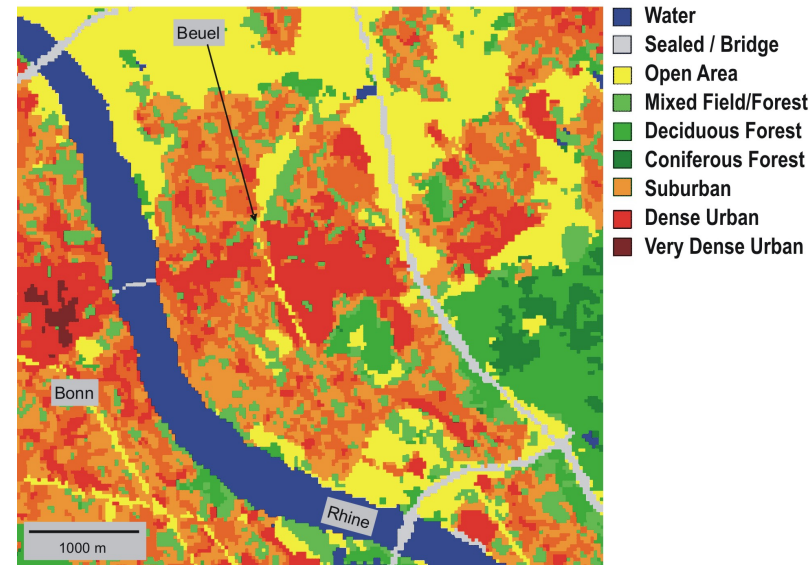

Fig. 1. Land usage of the regarded area. The land usage and 3$\mathrm{D}$ building data outside the black polygon is modified in order to simulate a single standing city (here the original data is shown).

a single standing city, the 3-D building data were removed outside of the polygon and the land usage was set to "Open Area" there. The remaining built-up area can be regarded as a typical medium size middle European city. The evaluated city area has a size of around $8 \mathrm{~km}^{2}$ with approx. 40000 inhabitants.

Five network topologies have been examined, which are referred to as scenario 1 to 5 . They can be divided into two general categories. In one, the sites are located within the urban area, and in the other category the sites are located outside the built-up area. Table 1 summarizes some characteristic information about the scenarios. For scenarios 3-5, two different antenna heights and varying distances to the built-up area are applied and analyzed in Sect. 3.1.

The base station antennas have a half power beam width of $33^{\circ}$ horizontal and $6.7^{\circ}$ vertical and $20.7 \mathrm{dBi}$ gain, except for scenario 2 , where antennas with $65^{\circ}$ horizontal and $6.7^{\circ}$ vertical half power beam width and $18 \mathrm{dBi}$ gain were applied. The power settings were identical for all scenarios: the maximum BS power per cell is $43 \mathrm{dBm}$, the constant $\mathrm{CPICH}$ power is $33 \mathrm{dBm}$ and the overall power for all other common channels is $36 \mathrm{dBm}$.

\subsection{Service and traffic assumptions}

The 3G simulations have been performed with a service mix consisting of four services as listed in Table 2 together with their main properties. The maximum allowed power to serve a single user in the downlink is $37 \mathrm{dBm}$ for all services. The mobiles are modeled as stationary in the dynamic simulation. For the services WWW and High data, an antenna gain of $2 \mathrm{dBi}$ (laptop device) is used, otherwise $0 \mathrm{dBi}$. The maximum active set size for the soft/softer handover algorithms is 3 . Table 2 also includes the average number of users who
Table 1. Overview of the 5 different network topologies.

\begin{tabular}{lcccc}
\hline Sites are ... & Scenario & Sites & $\begin{array}{c}\text { Sectors } \\
\text { per Site }\end{array}$ & $\begin{array}{c}\text { Antenna } \\
\text { height }\end{array}$ \\
\hline $\begin{array}{l}\text {.. inside } \\
\text { town }\end{array}$ & 1 & 1 & 6 & $40 \mathrm{~m}$ \\
\hline \multirow{2}{*}{ outside } & 2 & 7 & 3 & $25 \mathrm{~m}$ \\
town & 4 & 1 & 4 & $30 \mathrm{~m} / 60 \mathrm{~m}$ \\
& 5 & 2 & 4 & $30 \mathrm{~m} / 60 \mathrm{~m}$ \\
& & 5 & 3 & $30 \mathrm{~m} / 60 \mathrm{~m}$ \\
\hline
\end{tabular}

Table 2. Overview of the services applied in the $3 \mathrm{G}$ simulations for the reference traffic assumption $T_{1}$.

\begin{tabular}{lrrcc}
\hline \multirow{2}{*}{ Service } & \multicolumn{2}{c}{ Bitrate } & max. UL & active \\
& $\begin{array}{r}\text { DL } \\
\text { kbps }\end{array}$ & $\begin{array}{r}\text { UL } \\
\text { kbs }\end{array}$ & $\begin{array}{c}\text { power } \\
\text { dBm }\end{array}$ & UE \\
\hline Voice & 16 & 16 & 21 & 5.0 \\
Video Telephonie & 64 & 64 & 21 & 39.0 \\
WWW & 128 & 56 & 24 & 5.9 \\
High Data & 384 & 56 & 24 & 6.3 \\
\hline
\end{tabular}

simultaneously wish to use the corresponding service in the simulation area per service (offered traffic).

In order to determine a deterioration of the service quality with increasing network load, the simulations have been performed with a varying amount of offered traffic. A reference traffic assumption called $T_{1}$ - as given in Table $2-$ is scaled linearly, leading to traffic assumptions from $T_{0.25}\left(=T_{1}\right.$ multiplied with 0.25 ) up to $T_{3}$ (equals $T_{1}$ increased by a factor of $3)$.

In addition, the traffic is spatially distributed according to the land usage types, e.g. at land usage suburban, the offered traffic is half of that on the land usage dense urban. This leads to an inhomogeneous spatial traffic distribution, which yields a realistic modeling. The relative distribution of the active users over the services according to Table 2 is equal for all land usage types.

\section{Simulation results}

\subsection{Coverage}

Assuming the reference traffic assumption $T_{1}$ and the 384 kbps data service, Fig. 2 and Fig. 3 depict the coverage probability for scenario 2 and for scenario 5 , respectively.

The plots show very well that the network topology that uses sites outside the city leads to a restricted coverage especially of the city centre. This is due to the fact that especially 


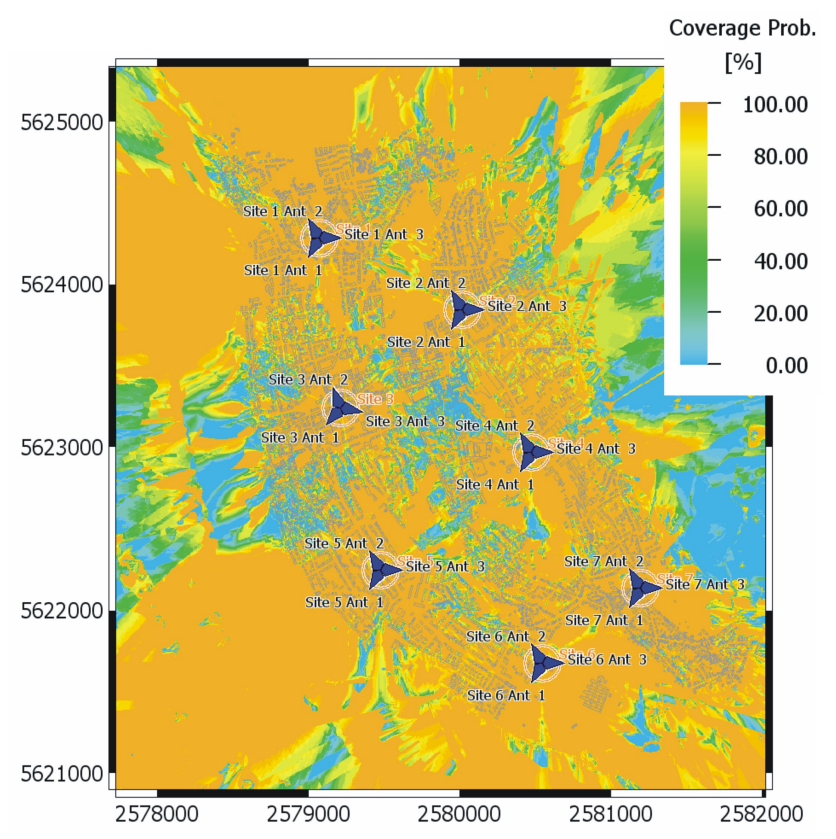

Fig. 2. Coverage probability for $384 \mathrm{kbps}$ data service for scenario 2 .

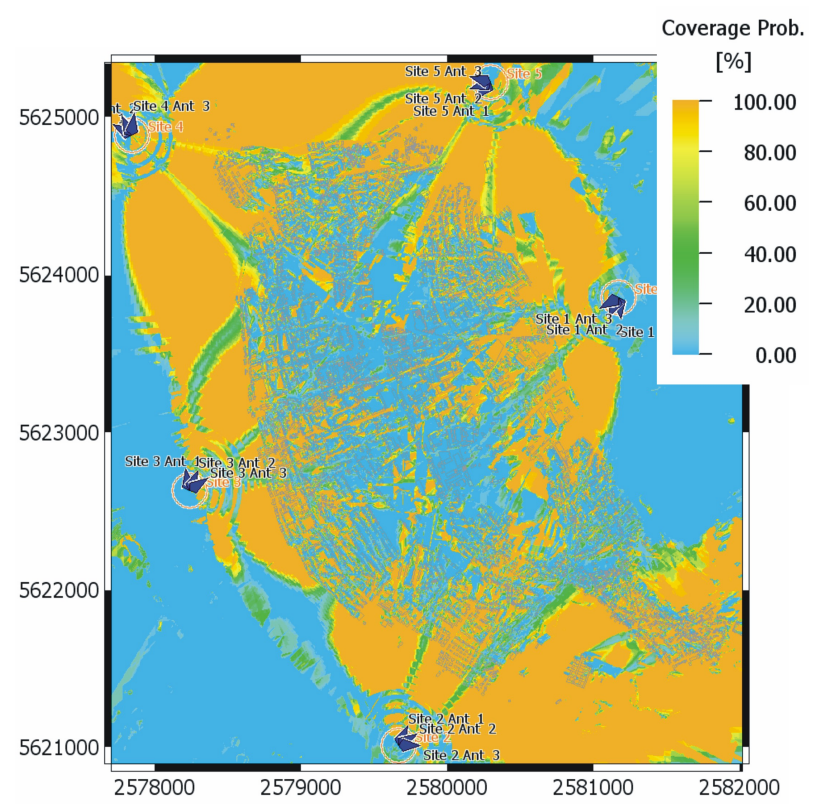

Fig. 3. Coverage probability for $384 \mathrm{kbps}$ data service for scenario 5 .

in the area in the middle between the base stations the cell isolation is very bad, leading to a poor interference situation.

As described in Sect. 2.2, the coverage plots represent a quasi-static evaluation. To account for shortages in the code resources of the network, the results should be interpreted in

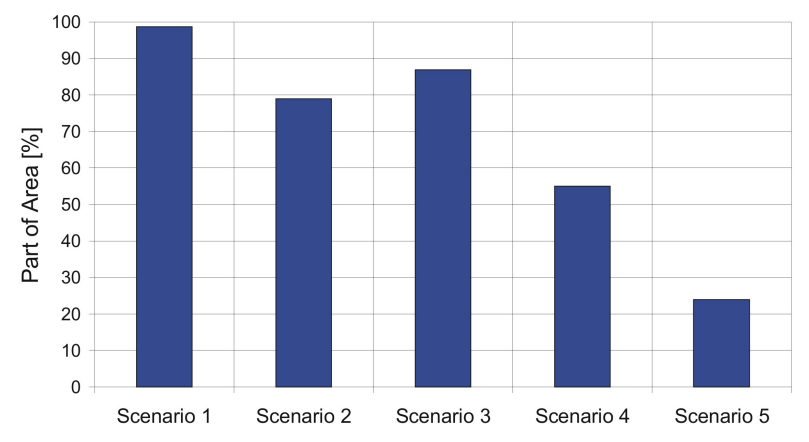

Fig. 4. Area with at least $90 \%$ coverage probability (384 kbps data service).

conjunction with the statistic results presented in Sect. 3.2 of this chapter.

Figure 4 shows the portion of the area that has a coverage probability of at least $90 \%$. This measure allows a good comparison between the scenarios.

Scenario 1 with its central base station is very advantageous in terms of the interference situation yielding excellent results. However, due to the low number of 6 sectors, the capacity of this scenario is limited (see Sect. 3.2).

When analyzing scenarios 3, 4 and 5 with sites outside the built-up area, it is noticeable that the coverage quality diminishes with a growing number of sectors. This reflects the growing interference problems with the increasing number of sectors located outside the city. On the other hand, in scenario 2 the different cell areas are better separated and the interference impact is thus more limited to the local environment. Therefore the overall interference level is comparably low, despite the high number of 21 sectors.

It should be mentioned that the coverage differences are less distinct for the other services (see Table 2), however high data rate services are one of the major reasons for using $3 \mathrm{G}$ networks.

When analyzing the resulting cell layout, the scenarios 3 , 4 and 5 lead to a much worse cell isolation than scenario 1 and 2 . The cell layout is more inhomogeneous in scenarios 3 to 5 , also leading to unfavorable handover conditions.

For the sites located outside the city, their distance to the built-up area was varied from 250 to $1000 \mathrm{~m}$, and the base station antenna heights were chosen to be 30 and $60 \mathrm{~m}$. There were no considerable differences in dependence of the distance; however, choosing a too short distance is disadvantageous, as the cells become more cluttered. The higher antenna heights turn out to be beneficial, as they lead to a better cell isolation when combined with higher antenna downtilt values. Therefore a distance of $500 \mathrm{~m}$ at $60 \mathrm{~m}$ antenna height is used for the detailed evaluation of scenarios 3,4 and 5 . 


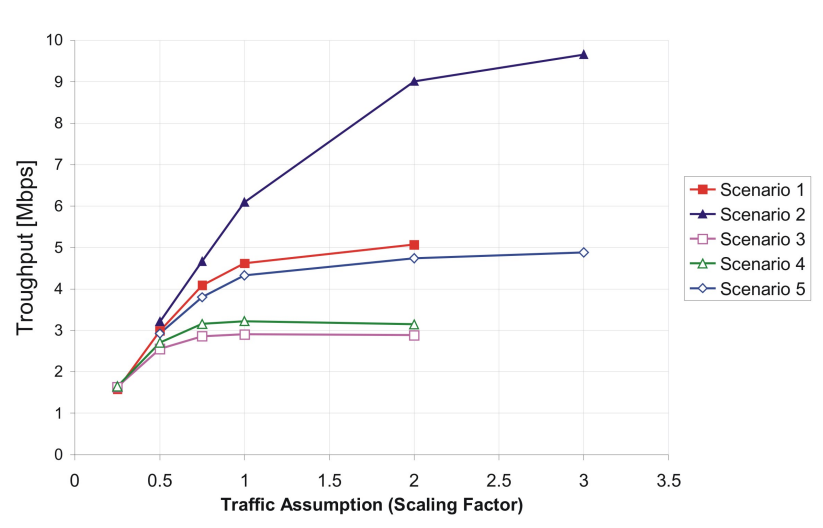

Fig. 5. Total throughput considering all services.

\subsection{Network capacity}

Figure 5 shows the total throughput for the whole network. As the number of sectors differs in the scenarios (see Table 1), higher total throughput values are expected if more sectors are available. The abscissa represents the scaled traffic (see Sect. 2.4). According to Fig. 5 scenario 2 yields the highest capacity, which is expected due to the high number of sectors. Scenario 5, which has 15 sectors, performs badly in terms of capacity, especially compared to scenario 1 with only 6 sectors. This reflects the poor interference situation in scenario 5 .

\subsection{Exposure}

For the following downlink exposure analysis the transmission power is derived as mean value from the dynamic simulation. A prediction of the received power has been performed for all base station sectors under consideration of the averaged transmission powers of the individual sectors. Finally, the propagation results were incoherently summed up in the whole simulation area, leading to the plots in Fig. 6 and Fig. 7.

Due to the increased radio channel attenuation if the sites are located outside the city, the exposure in the city is lower for scenario 5 compared to scenario 2 . On the other hand, the difference is less distinct as could be expected because of the fact that the required transmission powers are higher in scenario 5 (median values over all sectors: $24.4 \mathrm{dBm}$ for scenario 2, $27.8 \mathrm{dBm}$ for scenario 5).

Figure 8 shows a comparison between the exposure and the capacity for all five scenarios. The pink curve shows the total throughput in Mbps, the blue curve shows the $90 \%$ quantiles of the exposure values derived from results as shown in Fig. 6 and Fig. 7.

This comparison impressively shows that network topologies with base station sites outside the city yield the lowest

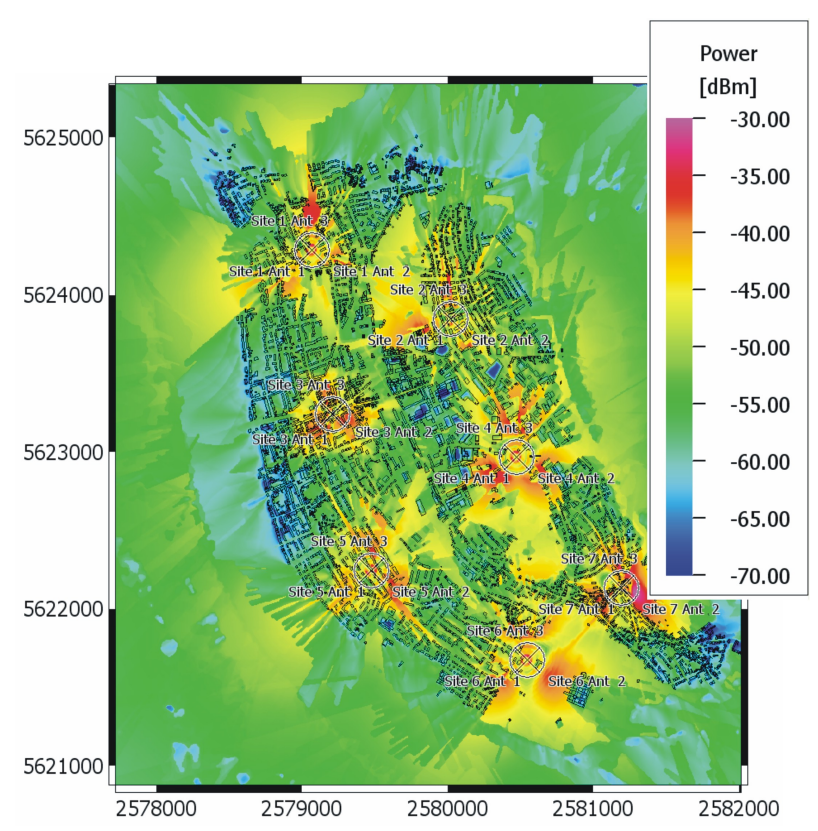

Fig. 6. Exposure for Scenario 2.

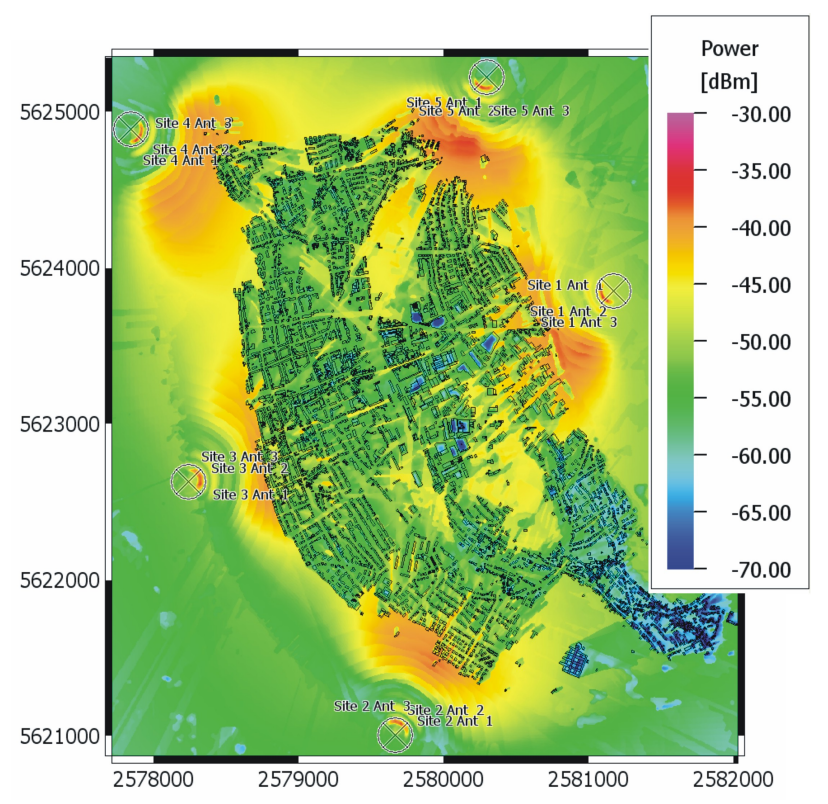

Fig. 7. Exposure for Scenario 5.

exposure, yet at the cost of a large reduction of the network capacity. Scenario 1 yields low exposure values at a reasonable network capacity. 


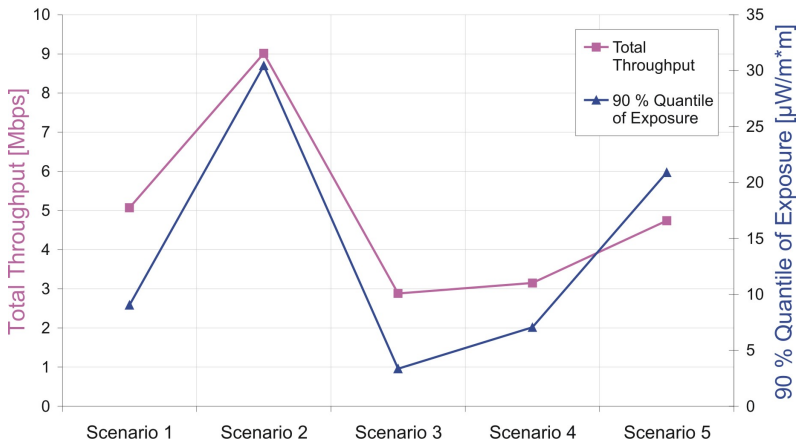

Fig. 8. Comparison of exposure and capacity for all scenarios.

\subsection{HSDPA and GSM/EDGE}

Furthermore simulations have been carried out investigating the impact of the different scenarios on HSDPA performance (Buddendick et al., 2004). It turned out that the mean expected data rate for a user in scenario 2 is twice as high as for a user in scenario 5.

In contrary, assuming that different carrier frequencies are assigned to all sectors, the coverage for GSM/EDGE systems obtained by scenario 5 is comparable to that of scenario 2 . Thus, concerning GSM/EDGE, it might be regarded as a realistic alternative to cover a medium size city with several sites located outside the city.

\section{Summary}

In this paper, five different network topologies were analyzed with regard to their capability to cover a medium size city with 3G mobile service. The impact of locating the sites inside or outside the city on coverage, capacity and exposure is examined. The simulations were performed with a dynamic $3 \mathrm{G}$ network simulator and a ray optical propagation model applying 3-D building data.

The scenarios with several sites located outside the city provide only unsatisfactory coverage for high data rate services. A service with $384 \mathrm{kbps}$ in the downlink is available in only $35 \%$ of the city area in case of five outer-city sites, but in $80 \%$ of the area in case of seven inner-city sites. However, for services with lower data rates all scenarios yield sufficient coverage in the complete city area.

A single outer-city site gives the lowest average exposure - which could be expected. However, scenarios with more than one outer-city sites yield average exposure values which exceed those of the scenario with only one site located in the city.
Furthermore, the required mobile transmission power to establish connections is lowest for the scenario with seven inner-city sites which leads to a low exposure of the active mobile users.

Regarding all three aspects (exposure, coverage and capacity), the scenarios with inner-city sites are in advantage. One outstanding site yields low exposure but insufficient capacity. Five outstanding sites yield insufficient coverage and the exposure is comparable to those of scenarios with inner-city sites. In addition, several outstanding sites lead to an inhomogeneous cell structure, which is generally of disadvantage.

However, one result of this paper is that a single inner-city site with medium antenna height $(40 \mathrm{~m})$ and six sectors yields a very good coverage and acceptable capacity in the city area at a low exposure. Thus, covering a medium-size city with only one or a few inner-city sites with a higher number of sectors is a topic of further investigations. Further work will also cover more detailed investigations on HSDPA network performance for different network topologies.

Acknowledgements. The authors would like to thank T-Mobile Germany for their initiation of the presented work and especially Dr. Beyer for the valuable help.

\section{References}

Rautiainen, T., Wölfle, G., and Hoppe, R.: Verifying Path Loss and Delay Spread Predictions of a 3D Ray Tracing Propagation Model in Urban Environments, 56th IEEE Vehicular Technology Conference (VTC) 2002 - Fall, Vancouver (British Columbia, Canada), Sept. 2002.

Zhang, Z., Yun, Z., Iskander, and Iskander, M.F.: Ray Tracing method for propagation models in wireless communication systems, IEE Electronics Lett., 36(5), 464-465, March 2000.

Wahl, R., Wölfle, G., Wertz, P., and Wildbolz, P.: "Dominant Path Prediction Model for Urban Scenarios", 14th IST Mobile and Wireless Communications Summit, Dresden (Germany), June 2005.

Heyn, T., Heuberger, A., and Keip, C.: Propagation Measurements for the Characterization of a Hybrid Mobile Channel in Sband, 14th IST Mobile Wireless Communications Summit 2005 - Dresden (Germany), June 2005.

Wertz, P., Hoppe, R., Wölfle, G., Buddendick, H., and Landstorfer, F.: Dynamic Simulator for the Analysis of the Performance of WCDMA Radio Networks, 4th COST 273 MCM-Meeting in Lisbon, Portugal, COST 273 TD(02)148, Sept. 2002.

Buddendick, H., Wölfle, G., Burger, S., and Wertz P.: Simulator for Performance Analysis in UMTS FDD Networks with HSDPA, 15th IEEE International Symposium on Personal, Indoor and Mobile Radio Communications (PIMRC) 2004, Barcelona (Spain), Sept. 2004.

WinProp, Models and software for Wave Propagation and Radio Network Planning, see http://www.awe-communications.com/, 2006. 